\title{
Low Glycemic Index Carbohydrates versus All Types of Carbohydrates for Treating Diabetes in Pregnancy: A Randomized Clinical Trial to Evaluate the Effect of Glycemic Control
}

\author{
Otilia Perichart-Perera, ${ }^{1}$ Margie Balas-Nakash,, ${ }^{1}$ Ameyalli Rodríguez-Cano, ${ }^{1}$ \\ Jennifer Legorreta-Legorreta, ${ }^{1}$ Adalberto Parra-Covarrubias, ${ }^{2}$ and Felipe Vadillo-Ortega ${ }^{3}$ \\ ${ }^{1}$ Nutrition Research Department, Instituto Nacional de Perinatología Isidro Espinosa de los Reyes, Montes Urales 800, \\ Lomas de Virreyes, 11000 Mexico City, Mexico \\ ${ }^{2}$ Endocrinology Department, Instituto Nacional de Perinatología Isidro Espinosa de los Reyes, Montes Urales 800, \\ Lomas de Virreyes, 11000 Mexico City, Mexico \\ ${ }^{3}$ Unidad de Vinculación, Facultad de Medicina, UNAM, Instituto Nacional de Medicina Genomica, \\ 04510 Mexico City, Mexico
}

Correspondence should be addressed to Otilia Perichart-Perera, o.perichart@inper.mx

Received 12 April 2012; Revised 16 October 2012; Accepted 17 October 2012

Academic Editor: Anil K. Agarwal

Copyright (C) 2012 Otilia Perichart-Perera et al. This is an open access article distributed under the Creative Commons Attribution License, which permits unrestricted use, distribution, and reproduction in any medium, provided the original work is properly cited.

\begin{abstract}
Background. Due to the higher prevalence of obesity and diabetes mellitus (DM), more pregnant women complicated with diabetes are in need of clinical care. Purpose. Compare the effect of including only low glycemic index (GI) carbohydrates (CHO) against all types of $\mathrm{CHO}$ on maternal glycemic control and on the maternal and newborn's nutritional status of women with type $2 \mathrm{DM}$ and gestational diabetes mellitus (GDM). Methods. Women ( $n=107, \leq 29$ weeks of gestation) were randomly assigned to one of two nutrition intervention groups: moderate energy and CHO restriction (Group 1: all types of CHO, Group 2: low GI foods). Results. No baseline differences in clinical data were observed. Capillary glucose concentrations throughout pregnancy were similar between groups. Fewer women in Group 2 exceeded weight gain recommendations. Higher risk of prematurity was observed in women in Group 2. No differences in glycemic control were observed between women with type 2 DM and those with GDM. Conclusions. Inclusion of low GI CHO as part of a comprehensive nutrition intervention is equally effective in improving glycemic control as compared to all types of $\mathrm{CHO}$. This strategy had a positive effect in preventing excessive maternal weight gain but increased the risk of prematurity.
\end{abstract}

\section{Introduction}

Diabetes mellitus (DM) is one of the leading causes of death among women in developing countries [1]. In Mexico, the prevalence of type $2 \mathrm{DM}$ has increased markedly [2], in parallel with a $50 \%$ increase prevalence of overweight and obesity [3]. The latter represents a major risk factor for increasing the risk of type $2 \mathrm{DM}$ and gestational diabetes (GDM) [4], associated with maternal and perinatal adverse outcomes [5,6], and long-term chronic diseases [7]. GDM prevalence in Mexico varies between 8-12\% [8,9].
Maternal, fetal, and neonatal adverse outcomes can be significantly reduced when blood glucose levels are maintained within normal ranges throughout pregnancy with medical and nutritional treatment [10]. Intensive diet therapy is recommended to achieve optimal glucose control, to meet the energy and nutrients needs during pregnancy, and to promote adequate weight gain. Medical nutrition therapy is recommended for all pregnant women with DM $[10,11]$. Carbohydrate $(\mathrm{CHO})$ restriction, $(40-45 \%$ of total energy intake (TEI)), moderate energy restriction (33\%), and capillary glucose self-monitoring appear to be essential 
for achieving glycemic control in these women [12, 13]. Nutrition interventions with dietary advice as part of the management have improved maternal and perinatal adverse outcomes, when compared to routine care [14, 15]. However, at present, it is not clear if different types of $\mathrm{CHO}$ in the diet may exert different effects; although decreasing the glycemic index (GI) of the diet appears to have an additional positive effect on glycemic control in patients with DM [16]. In a systematic review, a low GI diet in noncomplicated pregnant women decreased the birthweight and the frequency of largefor gestational-age (LGA) newborns, when compared to a higher GI diet [17]. A recent study in women with GDM showed a decrease in insulin needs when a low GI diet was prescribed compared to the American Diabetes Association diet [18]. No studies have described the effect of a low GI diet on glycemic control in women with GDM or previous DM. The aim of this study was to compare the effect of including only low GI CHO in the diet against all types of $\mathrm{CHO}$ on maternal glycemic control and on the maternal and newborn's nutritional status of women with type $2 \mathrm{DM}$ and GDM.

\section{Materials and Methods}

This paper reports the results of the two groups of pregnant women with DM who received a nutrition intervention within a randomized clinical trial (no. NCT00860613) conducted between 2004 and 2008. The Institutional Review Board of Instituto Nacional de Perinatología (Mexico City) approved the study protocol and the study was conducted according to the Declaration of Helsinki (as amended, October 2000).

Women were included if they had a gestational age $\leq 29$ weeks, had GDM or pregestational type $2 \mathrm{DM}$, and planned to attend their pregnancy in our institution. At that time, universal 2 step screening for GDM at $\geq 14$ weeks of gestation was done to all women. If the initial $50 \mathrm{~g}-1$ hour (h) glucose challenge test was $\geq 130 \mathrm{mg} / \mathrm{dL}(7.2 \mathrm{mmol} / \mathrm{dL})$, a $100 \mathrm{~g}-3 \mathrm{~h}$ oral glucose tolerance test was performed within two weeks and GDM was diagnosed with two or more abnormal values (fasting $\geq 95 \mathrm{mg} / \mathrm{dL}, 1 \mathrm{~h} \geq 180 \mathrm{mg} / \mathrm{dL}, 2 \mathrm{~h} \geq 155 \mathrm{mg} / \mathrm{dL}$, and $3 \mathrm{~h} \geq 140 \mathrm{mg} / \mathrm{dL}$ ) [11]. Women with type $2 \mathrm{DM}$ were diagnosed with a fasting glucose $>126 \mathrm{mg} / \mathrm{dL}$ in early pregnancy $(<14$ weeks) or a random glucose $>200 \mathrm{mg} / \mathrm{dL}$. If women were already treated for type $2 \mathrm{DM}$ before pregnancy, a new diagnosis was not made. Women were excluded if they had type $1 \mathrm{DM}$, renal, hepatic, or other metabolic diseases, or if they were unable to follow the nutrition intervention.

Women were enrolled by the staff of the Nutrition Department at the Endocrinology Outpatient Clinic. The protocol for recruitment included an initial visit in which suitability for randomization was evaluated, an invitation to participate in the study was done, and an informed consent was obtained. Women were randomly assigned (simple randomization) to the study groups (parallel design) by a clinical dietitian, using a random number list and sequentially numbered files. The dietitian was blinded to the allocation schedule.

\subsection{Intervention}

2.1.1. Group 1 (All Types of CHO). The intervention followed the American Dietetic Association nutrition practice guidelines for gestational diabetes [19]. Women received an individual food plan based on $\mathrm{CHO}$ restriction (40$45 \%$ of TEI), using a CHO counting strategy (basic level) [20]. Moderate energy restriction was recommended only for overweight and obese women $(24 \mathrm{kcal} / \mathrm{kg})$. Breakfast CHO intake was limited to $15-30 \mathrm{~g}$, and adequate fiber intake was promoted $(20-35 \mathrm{~g} /$ day $)$. Women in this group were advised to choose any type of $\mathrm{CHO}$, except added refined sugars.

Energy and $\mathrm{CHO}$ prescriptions were revised at every visit and changes were done according to weight gain and whether or not ketonuria was present. If ketones were present and weight gain was subnormal, energy prescription was increased (200 to $300 \mathrm{kcal} /$ day). If weight gain was adequate, energy was not modified and carbohydrates were increased (no more than $45 \%$ of TEI). Fat intake recommendation was maintained $(<40 \%$ of TEI), and protein recommendation adjustment was made accordingly $(20-25 \%$ of TEI).

2.1.2. Group 2 (Low GI CHO). Women in this group received the same intervention as women in Group 1, but were counseled to eliminate all moderate and high GI foods (GI > 55) [21]. Tropical fruits, refined breads, breakfast cereals, flour tortilla, white rice, refined cookies and pastries, potatoes, carrots, beets, and refined sugars were eliminated from their plan. Papaya was the only moderate GI fruit permitted because it is one of the most frequently consumed high-fiber foods in this population. Corn tortillas were included only when combined with beans, as well as corn flakes combined with milk, according to some evidence that the combination of these foods decreases their GI $[22,23]$.

Women in both groups received the same individual nutrition education at each visit, following a specific protocol designed for this study. Educational themes included the importance of healthy eating in DM, identification of $\mathrm{CHO}$, exchange lists and $\mathrm{CHO}$ counting, identification of high and low GI foods, healthy fats, and importance of capillary glucose self monitoring, among others. During each visit, concepts were reinforced, doubts were clarified, and women's skills in the application of the information provided were assessed (management of $\mathrm{CHO}$ servings, sample menus revision, etc.) assuring that all themes were covered during the intervention. Specific materials were designed for the educational purpose.

All women performed capillary glucose self-monitoring before and $2 \mathrm{~h}$ after meals ( 6 times/day, 2 days/week). Women were instructed on the use of the glucose meter and the recording of their glucose values in a specific format which included: time of the day, insulin dose, food intake, and glucose values before and $2 \mathrm{~h}$ after meals. At each visit, women reviewed their glucose monitoring records with the dietitian, identifying glucose values out of range and relating them with food intake. 
2.2. Clinical Followup. Women received the same routine obstetric prenatal care. Obstetricians, endocrinologists, and laboratory personnel were not aware of the study groups. No women were using antidiabetic therapy. The endocrinologist was responsible for prescribing human insulin (intermediate-acting and regular) twice a day plus prandial rapid-acting insulin analogs as needed to meet glucose goals.

Participants were followed-up every two to three weeks until the end of pregnancy. Nutrition baseline visits lasted 45 minutes, and followup visits were 30 minutes long. During each visit, nutrition assessment was done and nutrition recommendations were made accordingly.

Diet adherence was measured as the mean energy intake adequacy throughout the intervention and it was calculated based on the energy intake reported each visit and the energy intake recommendation from the previous visit (\% of energy adequacy $=$ energy intake/energy recommendation $\times 100$ ). Adequate adherence was considered to be $85-115 \%$.

\subsection{Primary Outcomes}

2.3.1. Maternal Glycemic Control. Fasting plasma glucose concentrations were measured every two weeks by a glucose oxidase method. At the beginning of the study, all women received a glucose meter (Medisense Optium, Abbott Laboratories, USA) and glucose blood strips (Medisense, Abbott Laboratories, USA) for pre- and $2 \mathrm{~h}$ postprandial selfmonitoring. Glucose goals were $\leq 95 \mathrm{mg} / \mathrm{dL}(5.27 \mathrm{mmol} / \mathrm{L})$ and $\leq 120 \mathrm{mg} / \mathrm{dL}(6.66 \mathrm{mmol} / \mathrm{L})$ for fasting and $2 \mathrm{~h}$ postprandial glucose, respectively. Insulin doses were reported by women in all visits.

\subsection{Secondary Outcomes}

2.4.1. Maternal Nutritional Status. Weight was measured with a calibrated digital scale (TANITA, Tokyo, Japan) to the nearest $\pm 0.1 \mathrm{~kg}$, while the subject was wearing light clothing and no shoes. Height was measured with a digital stadiometer (SECA, Germany) to the nearest $\pm 0.1 \mathrm{~cm}$. Pregestational weight was self-reported and was used to obtain the prepregnancy body mass index. Obesity classification was done according to the World Health Organization criteria [24]. Total weight gain was assessed according to the U.S.A. Institute of Medicine recommendations [25]. Excessive weight gain was defined as $>120 \%$ of recommended weight.

Diet was measured at baseline and every month (every 2 sessions) by the dietitian using the multiple-pass $24 \mathrm{~h}$ recall method. Food replicas were used to help with portion estimation. Nutrient analysis was performed using the Food Processor software (version 8.0, ESHA), which includes Mexican foods. Missing foods were added to the database using Mexican Tables of Nutritional Value [26]. The GI of the diet was calculated by multiplying the grams of $\mathrm{CHO}$ of each food consumed by the GI of that food, using glucose as standard, and adding up to a final number; that number was divided by the total grams of $\mathrm{CHO}$ consumed [27].
Women were instructed to collect their first morning urine sample in each visit and bring it to the dietitian. Ketones were measured with Multistix 10 SG (Bayer Diagnostics, Germany).

2.4.2. Newborn's Nutritional Status. Birthweight at birth was measured with a calibrated digital scale (TANITA 1582 Tokyo, Japan) to the nearest $\pm 0.1 \mathrm{~kg}$. Length was measured using an infantometer (SECA 207, Germany) to the nearest $\pm 0.1 \mathrm{~cm}$. Head circumference was measured with a flexible nonstretchable measuring tape (SECA 212, Germany).

Nutritional status was assessed in term newborns, using the WHO reference data [28]. Malnutrition was defined as -2 standard deviations (SD) (weight/length for wasting, length/age for stunting) and $<10$ percentile for head circumference/age. Newborns were classified as small for gestational age (SGA) (weight/age $<10$ percentile), LGA (weight/age $>90$ percentile), low birthweight (LBW) $(<2500 \mathrm{~g})$, or macrosomia ( $>4000 \mathrm{~g}$ ). For preterm newborns, the Babson reference data was used (SGA when weight/age $<10$ percentile, LGA $>90$ percentile) [29].

2.4.3. Adverse Clinical Outcomes. Preeclampsia was diagnosed according to our institution guidelines (blood pressure $>140 / 90 \mathrm{mmHg}$, and proteinuria $\geq 300 \mathrm{mg} / 24$ hours). Prematurity was considered when gestational age at birth was $<37$ weeks. These outcomes together with intrauterine and neonatal death were recorded from the medical chart.

2.5. Statistical Analysis. Sample size was estimated in order to obtain a difference of $10 \mathrm{mg} / \mathrm{dL}(\mathrm{sd} \pm 20 \mathrm{mg} / \mathrm{dL}$ ) in the decrease of glucose throughout the intervention, with an $80 \%$ power and an alpha of 0.05 , resulting in a minimum of 32 women in each group. All women were included in the analysis. Women lost to followup were assumed to maintain the last observation value (last observation carried forward imputation data) [30].

Fasting plasma glucose, insulin doses, and food intake were analyzed in three observation periods: a baseline (T1 ), a middle value (T-2), and a last visit value (T-3). T1 for capillary glucose measurements was obtained 2 weeks afterintervention. Ten women did not have any capillary glucose values; thus, sample size for the analysis of this variable was smaller $(n=97)$.

Descriptive statistics and frequencies for all variables were performed. Mean differences (Student's $t$-test or MannWhitney $U$-test for nonnormal distribution) were analyzed to assess correct randomization. Differences in proportions were done using chi-square and Fisher's exact test. Subgroup analyses were performed by type of DM. Intra and intergroup differences were assessed using repeated measures ANOVA, including study group and type of DM as factors. Multiple logistic regression was performed with type of DM and study group as independent variables and excessive maternal weight gain as dependent variable. Multiple logistic regression models were done for evaluating risk of adverse clinical outcomes. Type of DM, study group, presence of 
TABLE 1: Baseline personal, clinical, and glycemic data of pregnant women studied.

\begin{tabular}{|c|c|c|c|}
\hline$n=107$ & $\begin{array}{c}\text { Group } 1 \text { (All types of CHO) } \\
(n=46)\end{array}$ & $\begin{array}{c}\text { Group } 2 \text { (Low GI CHO) } \\
(n=61)\end{array}$ & $P$ value \\
\hline Age (years) ${ }^{\mathrm{a}}$ & $31.80 \pm 5.3$ & $32.30 \pm 4.8$ & 0.517 \\
\hline Type 2 DM $(\%, n)^{\mathrm{b}}$ & $50.0(23)$ & $52.50(32)$ & 0.856 \\
\hline $\operatorname{GDM}(\%, n)^{\mathrm{b}}$ & $50.0(23)$ & $47.50(29)$ & 0.789 \\
\hline Gestational age at admission (weeks) ${ }^{\mathrm{a}}$ & $20.70 \pm 6.7$ & $22.50 \pm 4.9$ & 0.182 \\
\hline Plasma fasting glucose $(\mathrm{mg} / \mathrm{dL})^{\mathrm{c}}$ & $104.10 \pm 31.83$ & $95.02 \pm 13.97$ & 0.406 \\
\hline Capillary fasting glucose $(\mathrm{mg} / \mathrm{dL})^{\mathrm{c}}$ & $96.00 \pm 13.91$ & $96.25 \pm 12.21$ & 0.531 \\
\hline Capillary $2 \mathrm{~h}$ postprandial glucose (breakfast) $(\mathrm{mg} / \mathrm{dL})^{\mathrm{a}}$ & $105.45 \pm 19.50$ & $108.76 \pm 19.10$ & 0.404 \\
\hline Capillary $2 \mathrm{~h}$ postprandial glucose (lunch) $(\mathrm{mg} / \mathrm{dL})^{\mathrm{a}}$ & $119.16 \pm 16.15$ & $118.79 \pm 21.98$ & 0.927 \\
\hline Capillary $2 \mathrm{~h}$ postprandial glucose (dinner) $(\mathrm{mg} / \mathrm{dL})^{\mathrm{a}}$ & $108.97 \pm 12.78$ & $116.20 \pm 21.96$ & 0.503 \\
\hline Insulin users $(\%, n)^{\mathrm{b}}$ & $69.60(32)$ & $56.70(34)$ & 0.192 \\
\hline Total insulin dose (units/day) ${ }^{\mathrm{c}}$ & $29.10 \pm 27.6$ & $19.80 \pm 22.5$ & 0.078 \\
\hline Years diagnosed with type 2 DM (years) ${ }^{c, d}$ & $3.75+2.33$ & $3.71+2.49$ & 0.870 \\
\hline Oral hypoglycemic agents used before pregnancy $(\%, n)^{\mathrm{b}, \mathrm{d}}$ & $81(17)$ & $85.7(24)$ & 0.905 \\
\hline
\end{tabular}

${ }^{\mathrm{a}}$ Mean $\pm \mathrm{SD}$ analyzed by Student $t$-test.

${ }^{b}$ Percentage $(n)$ analyzed by Chi square test.

${ }^{\mathrm{c}}$ Mean \pm SD analyzed by Mann-Whitney $U$ test.

${ }^{\mathrm{d}}$ Women with type $2 \mathrm{DM}(n=49)$.

TABLE 2: Baseline anthropometric and dietary data of pregnant women studied.

\begin{tabular}{|c|c|c|c|}
\hline$n=107$ & $\begin{array}{c}\text { Group } 1 \text { (All types of CHO) } \\
(n=46)\end{array}$ & $\begin{array}{l}\text { Group } 2 \text { (Low GI CHO) } \\
\qquad(n=61)\end{array}$ & $P$ value \\
\hline Weight $(\mathrm{kg})^{\mathrm{a}}$ & $73.95 \pm 16.59$ & $74.12 \pm 13.72$ & 0.953 \\
\hline Height $(\mathrm{cm})^{\mathrm{a}}$ & $152.36 \pm 6.92$ & $155.28 \pm 5.17$ & 0.054 \\
\hline Pregestational body mass index $\left(\mathrm{kg} / \mathrm{m}^{2}\right)^{\mathrm{a}}$ & $32.0 \pm 6.3$ & $30.50 \pm 5.2$ & 0.132 \\
\hline Overweight/obesity $(\%, n)^{\mathrm{b}}$ & $93.5(43)$ & $86.8(53)$ & 0.595 \\
\hline Energy intake $(\mathrm{kcal} / \text { day })^{\mathrm{c}}$ & $1525 \pm 479$ & $1535 \pm 560$ & 0.937 \\
\hline Carbohydrate intake $(\% \mathrm{TEI})^{\mathrm{c}}$ & $47.50 \pm 8.9$ & $50.50 \pm 8.6$ & 0.418 \\
\hline Protein intake $(\% \mathrm{TEI})^{\mathrm{a}}$ & $20.59 \pm 4.91$ & $19.59 \pm 5.26$ & 0.326 \\
\hline Lipid intake $(\% \mathrm{TEI})^{\mathrm{a}}$ & $32.09 \pm 8.41$ & $29.80 \pm 8.30$ & 0.166 \\
\hline Fiber intake $(\mathrm{g} / \text { day })^{\mathrm{a}}$ & $23.10 \pm 10.8$ & $24.30 \pm 12.8$ & 0.804 \\
\hline Saturated fat intake $(\% \mathrm{TEI})^{\mathrm{a}}$ & $10.00 \pm 3.20$ & $9.47 \pm 3.49$ & 0.433 \\
\hline Monounsaturated fat intake (\% TEI $)^{\mathrm{a}}$ & $9.85 \pm 4.31$ & $8.46 \pm 3.19$ & 0.060 \\
\hline Polyunsaturated fat intake $(\% \mathrm{TEI})^{\mathrm{c}}$ & $6.61 \pm 4.26$ & $5.17 \pm 3.25$ & 0.067 \\
\hline Glycemic index of diet ${ }^{\mathrm{a}}$ & $50.0 \pm 8.9$ & $51.20 \pm 7.2$ & 0.712 \\
\hline
\end{tabular}

${ }^{a}$ Mean \pm SD analyzed by Student $t$-test.

${ }^{b}$ Percentage $(n)$ analyzed by Chi square test.

${ }^{\mathrm{c}}$ Mean $\pm \mathrm{SD}$ analyzed by Mann-Whitney $U$ test.

$\% \mathrm{TEI}$ : total energy intake.

infection during pregnancy, and insulin use during pregnancy were the independent variables and clinical adverse outcomes were the dependent variables. A $P$ value $\leq 0.05$ was considered statistically significant. Statistics were done with the Statistical Package for the Social Sciences (SPSS) software, version 16.0 (Chicago, IL).

\section{Results}

We approached 766 women, but 618 did not meet the inclusion criteria and 41 refused to participate. From the total of women who met our eligibility criteria and agreed to participate $(n=107), 46$ women were assigned to the low glycemic index dietary strategy and 61 women to the dietary strategy with all types of $\mathrm{CHO}$ (Figure 1).

A total of 107 women received the nutrition intervention, 55 had type $2 \mathrm{DM}$ and 52 had GDM. Mean age was $32 \pm 5$ years (range: $20-42$ yrs) and mean gestational age at admission was $22 \pm 6$ weeks (range: 6 to 29 weeks). There were no baseline differences in personal, clinical, glycemic, anthropometric, and dietary data among the two groups (Tables 1 and 2). Pregestational overweight and obesity were 


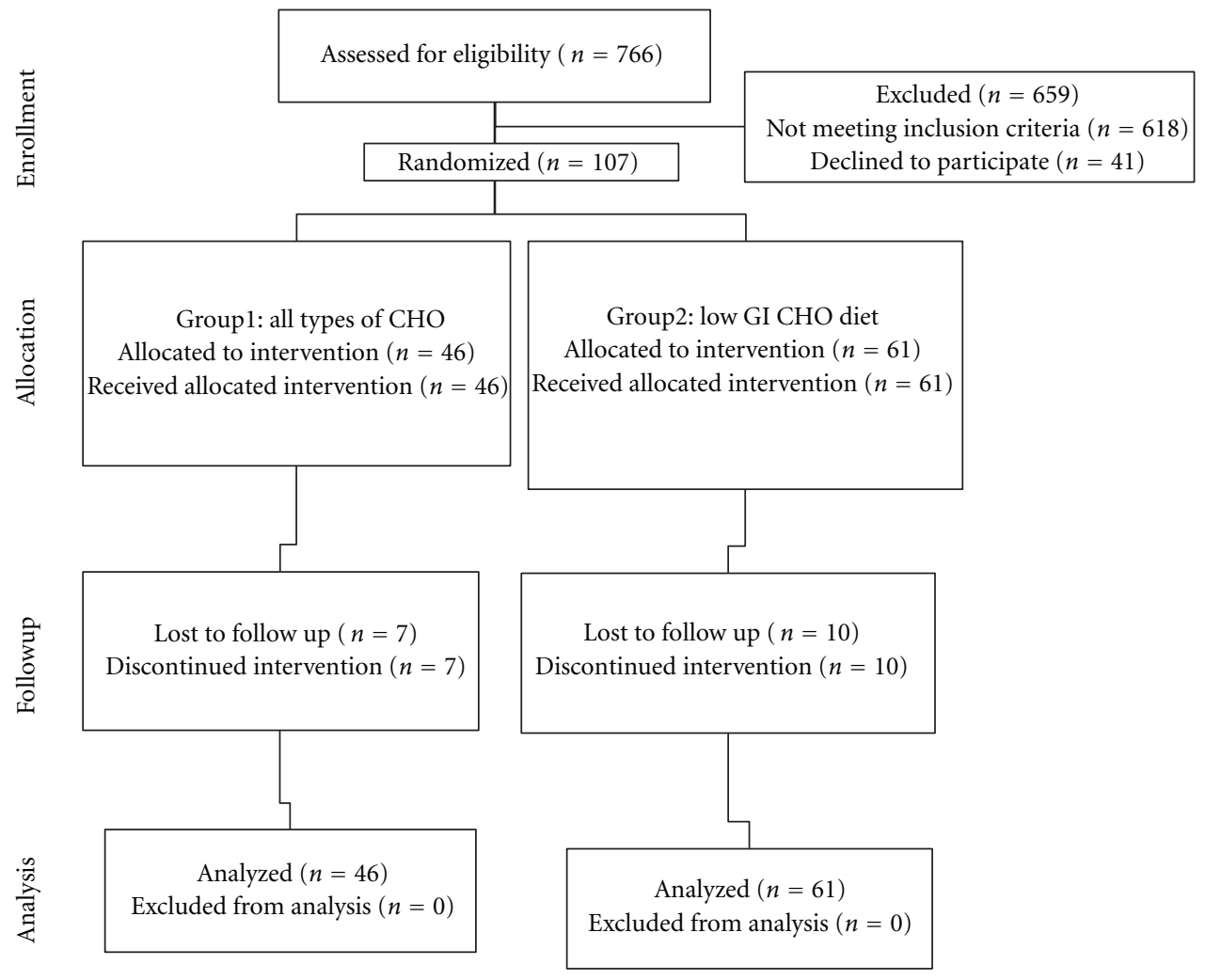

Figure 1: Flow diagram of trial.

present in $38.3 \%$ and $51.4 \%$ of women, respectively, without significant differences between groups.

Clinical followup was similar between groups. Women in Group 1 had a mean of $7.09 \pm 3.90$ visits with the dietitian throughout the intervention period, and women in Group 2 had $5.93 \pm 3.41(P>0.05)$. Overall, mean diet adherence was $83.05 \pm 12.57 \%$ and was not different between groups (Group $1: 80.75 \pm 11.62 \%$, Group 2: $86.15 \pm 18.08 ; P=0.108)$.

The proportion of women who discontinued the intervention $(n=17)$ was similar between the two groups (Group 1: $n=7$, Group 2: $n=10$ ). However, women were followed up until the end of pregnancy and were included analytically in the group to which they were randomized originally.

3.1. Primary Outcomes. Fasting plasma glucose decreased significantly in Group 1 and Group 2 throughout the intervention $(P<0.003$ and $P<0.004$, resp. $)$, but no significant differences were observed between groups. Capillary glucose concentrations throughout the intervention were similar among the two groups; the only significant decrease was observed in fasting capillary glucose (Table 3 ).

At the end of the intervention, the proportion of women who achieved glycemic goals at different mealtimes was similar between groups $(P>0.44)$. Women in Group 2 were successful in achieving glycemic goals at the end of pregnancy in $2 \mathrm{~h}$ postprandial glucose at lunch, preprandial, and $2 \mathrm{~h}$ postprandial glucose at dinner $(P<0.05)$. In Group 1 , the only significant increase in the proportion of women meeting capillary glycemic goals was observed after lunch $(P=0.03)$ (Table 4).

Even though there was a high variability in the number of visits women had, no differences in fasting or capillary glucose throughout the intervention were observed between women who started the intervention earlier $(<23$ weeks of gestation) and women who started later. The proportion of women achieving glycemic goals at the end of pregnancy was also similar regardless of the number of visits (data not shown).

The proportion of women who started using insulin during the intervention was similar between study groups in both types of DM ( $P \geq 0.40)$, without differences in insulin doses (Group 1: $42.7 \pm 26.4 \mathrm{u} / \mathrm{d}$ and Group 2: $35.5 \pm 21.5 \mathrm{u} / \mathrm{d}$ ) $(P=0.18)$.

As expected, more women with type $2 \mathrm{DM}$ used insulin during the intervention $(90.9 \%$ versus $31.4 \%, P<0.001$ ) and with higher doses $(41.02 \pm 21.9 \mathrm{u}$ /day versus $25.65 \pm$ 19.36 u/day, $P=0.030$ ) compared to women with GDM; however, no differences were observed by study groups in either type of DM.

3.2. Secondary Outcomes. Women with type $2 \mathrm{DM}$ gained more weight during pregnancy $(7.5 \pm 7.41 \mathrm{~kg})$ when compared to women with GDM $(4.35 \pm 6.59 \mathrm{~kg})(P=0.023)$; no differences were observed by study groups. Regardless of the type of DM, a lower proportion of women in Group 2 $(9.8 \%, n=6)$ was classified as having excessive weight gain 


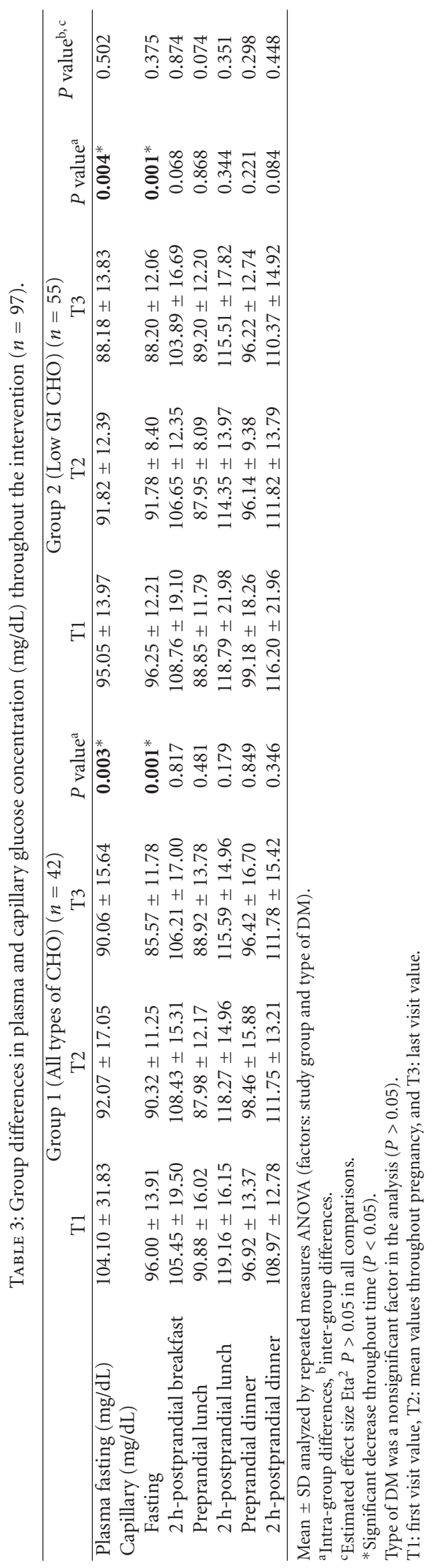


TABLE 4: Proportion of women who achieved glycemic goals in capillary blood before and after the intervention in both groups.

\begin{tabular}{|c|c|c|c|c|c|c|}
\hline \multirow{2}{*}{$n=97$} & \multicolumn{3}{|c|}{ Group 1 (All types of CHO) $(n=42)$} & \multicolumn{3}{|c|}{ Group 2 (Low GI CHO) $(n=55)$} \\
\hline & Baseline (\%) & Final $(\%)$ & $P$ value ${ }^{\mathrm{a}}$ & Baseline (\%) & Final $(\%)$ & $P$ value ${ }^{\mathrm{a}}$ \\
\hline Fasting (mg/dL) & 59.5 & 90.5 & 0.139 & 49.1 & 81.8 & 0.078 \\
\hline $2 \mathrm{~h}$-postprandial breakfast (mg/dL) & 78.6 & 88.1 & 0.281 & 83.6 & 92.7 & 0.121 \\
\hline Preprandial lunch (mg/dL) & 61.9 & 77.8 & 0.063 & 79.6 & 80.6 & 0.693 \\
\hline $2 \mathrm{~h}$-postprandial lunch (mg/dL) & 52.4 & 61.9 & 0.031 & 64.8 & 70.4 & 0.035 \\
\hline Preprandial dinner $(\mathrm{mg} / \mathrm{dL})$ & 42.9 & 57.1 & 0.087 & 50.0 & 57.4 & 0.001 \\
\hline $2 \mathrm{~h}$-postprandial dinner $(\mathrm{mg} / \mathrm{dL})$ & 78.6 & 83.3 & 0.614 & 66.7 & 77.8 & 0.037 \\
\hline
\end{tabular}

${ }^{a}$ Within-group difference in the proportion of women analyzed with chi-square test.

compared to women in Group $1(34.8 \%, n=16)(P=$ 0.002) (RR: $3.53,95 \%$ CI 1.50 to 8.32 ).

Energy and macronutrient intakes throughout pregnancy are described in Table 5. There was a significant decrease in the GI of the diet throughout the intervention period only in Group $2(51.29 \pm 7.28$ to $47.18 \pm 6.93, P=$ $0.001)$. No other significant dietary differences were observed between study groups.

A trend for lower birthweight among newborns in Group 2 was observed $(2883.9 \pm 676.8 \mathrm{~g}$ versus $3115.5 \pm 534.8 \mathrm{~g})(P=$ 0.06 ) (95\% CI of the difference: -13.55 to 476.72 ). The rate of macrosomia (Group 1: 6.8\% versus Group 2: 3.4\%, $P=$ 0.649 ) and low birthweight (Group 1: $18.6 \%$ versus Group 2: $9.1 \%, P=0.140$ ) were similar between study groups. No inter-group differences in the frequency of SGA (Group 1: $6.8 \%$ versus Group 2: 10.2\%) or LGA (Group 1: $4.5 \%$ versus Group 2: $5.1 \%$ ) were detected. Wasting was present in $7.7 \%$ (Group 1) and 6.5\% (Group 2) and stunting was observed in $4.5 \%$ (Group 1) and $11.9 \%$ (Group 2), without showing significant inter-group differences. No newborns from Group 1 had low head circumference, while 1 newborn in Group 2 was classified as having low head circumference $(P \geq 0.05)$. No differences by the type of DM were observed in any newborn nutritional marker.

No differences were observed between groups in the frequency of the clinical adverse outcomes studied $(P>$ $0.05)$, but a trend of a higher frequency of prematurity was observed in Group 2 (19\% versus $11.3 \%, P=0.237$ ). No differences were found in these clinical outcomes between women with GDM and type $2 \mathrm{DM}$ within each study group $(P>0.05)$.

Multivariate analysis showed the same risk of preeclampsia, intrauterine, and neonatal death in women from both study groups, but a higher risk of prematurity in women in Group 2 (RR: $4.74,95 \%$ CI: 1.08 to $20.84, P=0.03$ ). The model was adjusted by type of DM, insulin use, and presence of infection during pregnancy.

\section{Discussion}

Nutrition intervention has been recognized as the cornerstone treatment for achieving glycemic control in pregnant women with DM $[11,19]$, and the general dietary guidelines for GDM treatment include $\mathrm{CHO}$ restriction and the promotion of adequate fiber and healthy fat intake [19]. No previous evidence exists regarding the effect of lowering the GI of the diet on glycemic control in pregnant women with type $2 \mathrm{DM}$ or GDM. This study shows that including only low GI $\mathrm{CHO}$ in the diet is associated with similar glucose concentrations throughout pregnancy, when compared to women following the same energy and macronutrient prescription (40-45\% of $\mathrm{CHO}, 20-25 \mathrm{~g}$ of fiber), but with moderate and high GI foods. However, as previously reported $[11,31,32]$, the nutrition intervention had a positive effect in decreasing fasting plasma and capillary glucose throughout pregnancy in both groups and both types of diabetes. The proportion of women who achieved glycemic goals at the end of the intervention also increased at some mealtimes. Even though, there were no inter-group differences in capillary glucose, women in Group 2 achieved glycemic control at more mealtimes than women in Group 1 (intra-group comparisons). These findings are clinically important considering that the main goal of DM treatment in pregnancy is to achieve a consistent glycemic control throughout the day, and achieving postprandial glycemic control has been identified as a main factor for reducing DM-related complications [33].

A main strength of this study is that it provides data about the effect of low GI CHO on glycemic control and shows that this effect is not different between women with GDM and women with type $2 \mathrm{DM}$.

No differences in insulin users or doses were observed by study groups. In a recent study, fewer women with GDM, who were receiving a low GI diet, needed insulin during pregnancy, when compared to the American Diabetes Association diet [18]. Our results are not comparable, because in that study, a strict protocol to start women on insulin was followed. Insulin prescription was based on inadequate glycemic control and depended on the clinical judgment of the endocrinologist.

Fewer women in the low GI group gained excessive weight, according to IOM guidelines [25]. This could be relevant because most women in our study were overweight or obese $(90 \%)$, and it is well known that prepregnancy obesity and excessive gestational weight gain may increase the frequency of adverse pregnancy outcomes (preeclampsia, cesarean delivery, LGA, macrosomia, and/or neonatal adiposity) $[34,35]$.

Similar to a previous report [36], a trend to lower but within normal birthweight was observed in newborns of women in the low GI diet group, without increasing the frequency of SGA or LBW. Birthweight and weight indices 
TABLE 5: Dietary intake throughout the intervention in both study groups.

\begin{tabular}{|c|c|c|c|c|c|c|c|c|c|}
\hline \multirow{2}{*}{$n=107$} & \multicolumn{4}{|c|}{ Group 1 (All types of CHO) $(n=46)$} & \multicolumn{4}{|c|}{ Group 2 (Low GI CHO) $(n=61)$} & \multirow{2}{*}{$P$ value $^{\mathrm{b}, \mathrm{c}}$} \\
\hline & $\mathrm{T} 1$ & $\mathrm{~T} 2$ & T3 & $P$ value ${ }^{\mathrm{a}}$ & $\mathrm{T} 1$ & $\mathrm{~T} 2$ & T3 & $P$ value $^{\mathrm{a}}$ & \\
\hline Energy (kcal) & $1525 \pm 479$ & $1568 \pm 478$ & $1613 \pm 474$ & 0.299 & $1542 \pm 566$ & $1507 \pm 379$ & $1506 \pm 438$ & 0.697 & 0.838 \\
\hline Protein (\% TEI) & $20.58 \pm 4.91$ & $21.81 \pm 4.79$ & $21.15 \pm 5.23$ & 0.596 & $19.83 \pm 5.45$ & $23.54 \pm 20.15$ & $21.45 \pm 4.42$ & 0.058 & 0.582 \\
\hline $\begin{array}{l}\text { Carbohydrates ( } \% \\
\text { TEI) }\end{array}$ & $47.50 \pm 8.95$ & $47.88 \pm 6.84$ & $45.78 \pm 8.27$ & 0.333 & $48.67 \pm 8.61$ & $47.59 \pm 8.17$ & $46.59 \pm 9.09$ & 0.957 & 0.530 \\
\hline Fiber (g/day) & $23.11 \pm 10.84$ & $25.99 \pm 11.55$ & $20.58 \pm 4.91$ & 0.211 & $24.70 \pm 12.84$ & $26.81 \pm 10.78$ & $25.36 \pm 10.31$ & 0.632 & 0.666 \\
\hline Lipids (\% TEI) & $32.08 \pm 8.41$ & $30.26 \pm 6.75$ & $32.47 \pm 7.36$ & 0.800 & $29.45 \pm 8.37$ & $28.38 \pm 9.96$ & $27.90 \pm 8.51$ & 0.300 & 0.518 \\
\hline Saturated fat (\% TEI) & $10.00 \pm 3.26$ & $10.43 \pm 3.20$ & $10.60 \pm 2.96$ & 0.262 & $9.31 \pm 3.56$ & $8.51 \pm 2.90$ & $8.70 \pm 3.27$ & 0.326 & 0.473 \\
\hline $\begin{array}{l}\text { Monounsaturated fat } \\
(\% \text { TEI })\end{array}$ & $9.84 \pm 4.31$ & $8.86 \pm 2.74$ & $8.86 \pm 2.74$ & 0.804 & $8.27 \pm 3.29$ & $8.31 \pm 6.17$ & $7.91 \pm 3.23$ & 0.521 & 0.114 \\
\hline $\begin{array}{l}\text { Polyunsaturated fat } \\
(\% \mathrm{TEI})\end{array}$ & $6.95 \pm 3.97$ & $5.06 \pm 3.21$ & $6.00 \pm 4.11$ & 0.190 & $5.49 \pm 4.01$ & $5.62 \pm 4.96$ & $4.96 \pm 3.09$ & 0.385 & 0.475 \\
\hline Glycemic index & $50.00 \pm 8.98$ & - & $48.61 \pm 8.37$ & 0.345 & $51.29 \pm 7.28$ & - & $47.18 \pm 6.93$ & $0.001^{*}$ & 0.921 \\
\hline
\end{tabular}

Mean \pm SD analyzed by repeated measures ANOVA.

${ }^{a}$ Intra-group differences, ${ }^{b}$ inter-group differences.

cEstimated effect size $\mathrm{Eta}^{2} P>0.05$ in all comparisons.

* Significant decrease throughout time $(P<0.05)$.

T1: first visit value, T2: mean values during pregnancy, and T3: last visit value.

\%TEI: Total energy intake.

have been used as indirect markers of intrauterine nutrition and fetal programming $[37,38]$ and the promotion of a healthy birthweight may be an important goal when treating obesity and DM in pregnancy. However, the implications that these findings may have in decreasing the short- and long-term nutritional and metabolic risks of the newborn are unknown.

A relevant finding was the higher risk of prematurity observed in women who followed the low GI dietary recommendations. This observation has not been reported previously, but there is evidence showing that dietary restriction during pregnancy is associated with higher risk of prematurity (OR: 1.14, 95\% CI: 1.03 a 1.25, $P=0.009$ ) [39]. The restriction of moderate and high GI foods in Group 2 may have resulted in a more restricted diet that could be associated with prematurity as well as with the observed lower frequency of excessive weight gain and the trend of lower birthweight in women in this group. However, our dietary data does not show any differences in energy intake between groups.

Our study has some limitations that should be discussed. Firstly, bias may have been introduced by the fact that capillary glucose concentrations were self-recorded because of the lack of memory capacity of the glucose meters used [40]. Besides, baseline capillary glucose values were not available, since women received dietary and self-monitoring recommendations during the first prenatal visit, so the first capillary glucose value was already a result of the dietary intervention. It is likely that at the beginning of the study period (from the first to the second visit) a drop in capillary glucose values may have occurred, masking the expected intra-group decrease throughout pregnancy with nutrition interventions. In addition, insulin data was obtained from the medical prescription, and we assumed that women applied their insulin dose as prescribed. Secondly, a systematic error was introduced in the calculation of the diet GI due to the lack of GI data of some Mexican foods and food combinations. Finally, while the 24-hourrecall is useful in indirectly evaluating adherence to the nutrition intervention, the method has some limitations, including relying on the patient's memory, not evaluating usual energy and macronutrient intake, and the possibility of falsely reporting compliance with the intervention guidelines during an ongoing nutrition education process [41].

In conclusion, the inclusion of low GI CHO as part of a comprehensive nutrition intervention was equally effective in improving glycemic control as compared to all types of $\mathrm{CHO}$, without differences between pregnant women with type $2 \mathrm{DM}$ and women with GDM. The low GI strategy had a positive effect in preventing excessive maternal weight gain, but women in this group had higher risk of preterm birth. More data is needed before using the recommendation of restricting high and moderate GI foods for treating DM in pregnancy as a better strategy than including all types of $\mathrm{CHO}$. Future research should evaluate the effects of a low GI diet on the risk of later developing type $2 \mathrm{DM}$ in women with GDM and on newborn and child adiposity and metabolic markers.

\section{Conflict of Interests}

The authors declare that there is no conflict of interests.

\section{Acknowledgments}

The authors would like to thank Paola Alonso de la Vega, Carlos Ortega González, and Ma. Aurora Ramírez Torres for their support. 


\section{References}

[1] Mexican Ministry of Health, National Registry of Health information, 2011, http://www.sinais.salud.gob.mx/mortalidad/.

[2] J. A. Rull, C. A. Aguilar-Salinas, R. Rojas, J. M. Rios-Torres, F. J. Gómez-Pérez, and G. Olaiz, "Epidemiology of type 2 diabetes in Mexico," Archives of Medical Research, vol. 36, no. 3, pp. 188-196, 2005.

[3] G. Olaiz-Fernández, J. Rivera-Dommarco, T. Shamah-Levy et al., National Health and Nutrition Survey 2006, 2006.

[4] A. Ramirez-Torres, "Diabetes mellitus gestacional. Experience in a third level institution," Ginecología y Obstetricia de México, vol. 73, pp. 484-491, 2005.

[5] J. M. Baeten, E. A. Bukusi, and M. Lambe, "Pregnancy complications and outcomes among overweight and obese nulliparous women," American Journal of Public Health, vol. 91, no. 3, pp. 436-440, 2001.

[6] E. Burstein, A. Levy, M. Mazor, A. Wiznitzer, and E. Sheiner, "Pregnancy outcome among obese women: a prospective study," American Journal of Perinatology, vol. 25, no. 9, pp. 561-566, 2008.

[7] E. A. Reece, "The fetal and maternal consequences of gestational diabetes mellitus," Journal of Maternal-Fetal and Neonatal Medicine, vol. 23, no. 3, pp. 199-203, 2010.

[8] A. Espinosa De Los Monteros, A. Parra, R. Hidalgo, and M. Zambrana, "The after breakfast 50-g, 1-hour glucose challenge test in urban Mexican pregnant women: its sensitivity and specificity evaluated by three diagnostic criteria for gestational diabetes mellitus," Acta Obstetricia et Gynecologica Scandinavica, vol. 78, no. 4, pp. 294-298, 1999.

[9] G. Forsbach, C. Cantú-Diaz, J. Vázquez-Lara, M. A. Villanueva-Cuellar, C. Alvarez y García, and E. RodríguezRamírez, "Gestational diabetes mellitus and glucose intolerance in a Mexican population," International Journal of Gynecology and Obstetrics, vol. 59, no. 3, pp. 229-232, 1997.

[10] L. Jovanovic, "Medical nutritional therapy in pregnant women with pregestational diabetes mellitus," Journal of MaternalFetal and Neonatal Medicine, vol. 9, no. 1, pp. 21-28, 2000.

[11] American Diabetes Association, "Position statement: gestational diabetes mellitus," Diabetes Care, vol. 27, pp. 88-90, 2004.

[12] A. Dornhorst and G. Frost, "The principles of dietary management of gestational diabetes: reflection on current evidence," Journal of Human Nutrition and Dietetics, vol. 15, no. 2, pp. 145-156, 2002.

[13] R. H. Knopp, M. S. Magee, V. Raisys, T. Benedetti, and B. Bonet, "Hypocaloric diets and ketogenesis in the management of obese gestational diabetic women," Journal of the American College of Nutrition, vol. 10, no. 6, pp. 649-667, 1991.

[14] C. A. Crowther, J. E. Hiller, J. R. Moss, A. J. McPhee, W. S. Jeffries, and J. S. Robinson, "Effect of treatment of gestational diabetes mellitus on pregnancy outcomes," The New England Journal of Medicine, vol. 352, no. 24, pp. 2477-2486, 2005.

[15] M. B. Landon, C. Y. Spong, E. Thom et al., "A multicenter, randomized trial of treatment for mild gestational diabetes," The New England Journal of Medicine, vol. 361, no. 14, pp. 1339-1348, 2009.

[16] D. Thomas and E. J. Elliott, "Low glycaemic index, or low glycaemic load, diets for diabetes mellitus," Cochrane Database of Systematic Reviews, no. 1, Article ID CD006296, 2009.

[17] J. Tieu, C. A. Crowther, and P. Middleton, "Dietary advice in pregnancy for preventing gestational diabetes mellitus," Cochrane Database of Systematic Reviews, no. 2, Article ID CD006674, 2008.
[18] R. G. Moses, M. Barker, M. Winter, P. Petocz, and J. C. BrandMiller, "Can a low-glycemic index diet reduce the need for insulin in gestational diabetes mellitus? A randomized trial," Diabetes Care, vol. 32, no. 6, pp. 996-1000, 2009.

[19] American Dietetic Association, Medical Nutrition Therapy: Nutrition Practice Guidelines for Gestational Diabetes, American Dietetic Association, Chicago, Ill, USA, 2001.

[20] S. J. Gillespie, K. D. Kulkarni, and A. E. Daly, "Using carbohydrate counting in diabetes clinical practice," Journal of the American Dietetic Association, vol. 98, no. 8, pp. 897-905, 1998.

[21] K. Foster-Powell, S. H. A. Holt, and J. C. Brand-Miller, "International table of gylcemic index and glycemic load values: 2002," American Journal of Clinical Nutrition, vol. 76, no. 1, pp. 5-56, 2002.

[22] A. C. Frati-Munari, R. A. Roca-Vides, R. J. López-Pérez, I. de Vivero, and M. Ruiz-Velazco, "The glycemic index of some foods common in Mexico," Gaceta Medica de Mexico, vol. 127, no. 2, pp. 163-170, 1991

[23] M. Sugiyama, A. C. Tang, Y. Wakaki, and W. Koyama, "Glycemic index of single and mixed meal foods among common Japanese foods with white rice as a reference food," European Journal of Clinical Nutrition, vol. 57, no. 6, pp. 743752,2003

[24] World Health Organization, "Diet, nutrition and the prevention of chronic diseases," WHO Technical Report Series, World Health Organization, Geneva, Switzerland, 2003.

[25] K. Rasmussen and A. Yaktine, Weight Gain during Pregnancy: Reexamining the Guidelines. Institute of Medicine and National Research Council, National Academy Press, Washington, DC, USA, 2009.

[26] M. Chávez, M. Hernández, and J. Roldán, Tablas de uso práctico del valor nutrimental de los alimentos de mayor consumo en México, Instituto Nacional de Nutrición Salvador Zubirán, Mexico City, Mexico, 1992.

[27] T. M. S. Wolever, P. M. Nguyen, J. L. Chiasson et al., "Determinants of diet glycemic index calculated retrospectively from diet records of 342 individuals with non-insulin-dependent diabetes mellitus," American Journal of Clinical Nutrition, vol. 59, no. 6, pp. 1265-1269, 1994.

[28] World Health Organization, WHO Child Growth Standards: Length/Height for age, Weight for age, Weight for length, weight for height, and body mass index for age. Methods and Development, 2006.

[29] T. R. Fenton, "A new growth chart for preterm babies: babson and Benda's chart updated with recent data and a new format," BMC Pediatrics, vol. 3, article 13, 2003.

[30] J. M. Engels and P. Diehr, "Imputation of missing longitudinal data: a comparison of methods," Journal of Clinical Epidemiology, vol. 56, no. 10, pp. 968-976, 2003.

[31] C. A. Major, M. J. Henry, M. De Veciana, and M. A. Morgan, "The effects of carbohydrate restriction in patients with dietcontrolled gestational diabetes," Obstetrics and Gynecology, vol. 91, no. 4, pp. 600-604, 1998.

[32] D. Reader, P. Splett, and E. P. Gunderson, "Impact of gestational diabetes mellitus nutrition practice guidelines implemented by registered dietitians on pregnancy outcomes," Journal of the American Dietetic Association, vol. 106, no. 9, pp. 1426-1433, 2006.

[33] L. G. Jovanovic, "Using meal-based self-monitoring of blood glucose as a tool to improve outcomes in pregnancy complicated by diabetes," Endocrine Practice, vol. 14, no. 2, pp. 239247,2008 
[34] A. M. Siega-Riz, A. M. Siega-Riz, and B. Laraia, "The implications of maternal overweight and obesity on the course of pregnancy and birth outcomes," Maternal and Child Health Journal, vol. 10, no. 5, supplement, pp. S153-S156, 2006.

[35] N. J. Sebire, M. Jolly, J. P. Harris et al., "Maternal obesity and pregnancy outcome: a study of 287213 pregnancies in London," International Journal of Obesity, vol. 25, no. 8, pp. 1175-1182, 2001.

[36] R. G. Moses, M. Luebcke, W. S. Davis et al., "Effect of a lowglycemic-index diet during pregnancy on obstetric outcomes," American Journal of Clinical Nutrition, vol. 84, no. 4, pp. 807812, 2006.

[37] A. J. Drake and B. R. Walker, "The intergenerational effects of fetal programming: non-genomic mechanisms for the inheritance of low birth weight and cardiovascular risk," Journal of Endocrinology, vol. 180, no. 1, pp. 1-16, 2004.

[38] H. Delisle, Programming of Chronic Disease by Impaired Fetal Nutrition. Evidence and Implications for Policy and Intervention Strategies, World Health Organization, Geneva, Switzerland, 2002.

[39] P. Haggarty, D. M. Campbell, S. Duthie et al., "Diet and deprivation in pregnancy," British Journal of Nutrition, vol. 102, no. 10, pp. 1487-1497, 2009.

[40] C. J. Homko and E. A. Reece, "Self-monitoring of blood glucose in gestational diabetes," Journal of Maternal-Fetal and Neonatal Medicine, vol. 12, no. 6, pp. 389-395, 2002.

[41] I. M. Buzzard, C. L. Faucett, R. W. Jeffery et al., "Monitoring dietary change in a low-fat diet intervention study: advantages of using 24-hour dietary recalls vs food records," Journal of the American Dietetic Association, vol. 96, no. 6, pp. 574-579, 1996. 


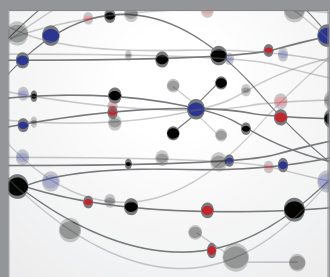

The Scientific World Journal
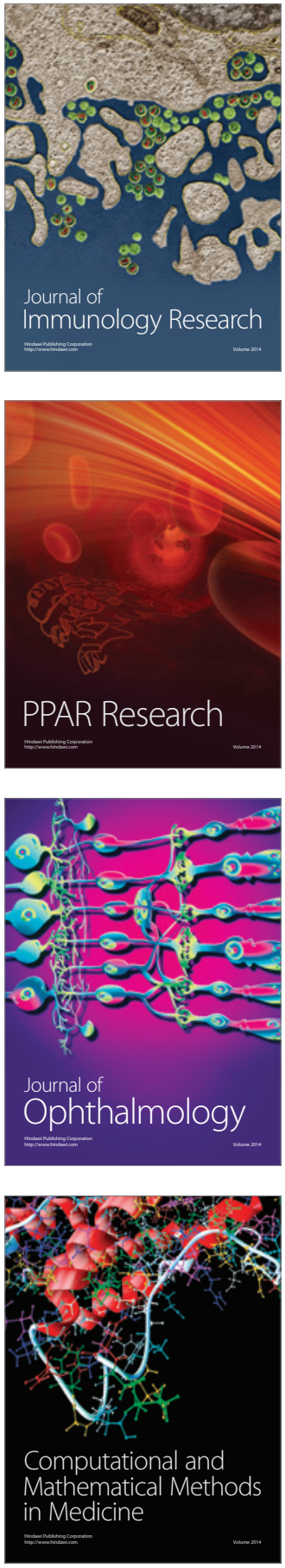

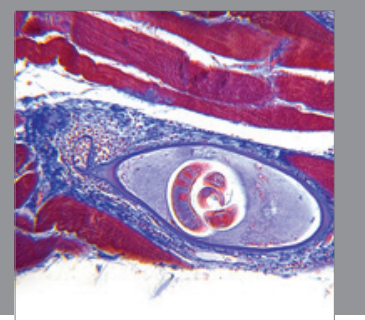

Gastroenterology

Research and Practice
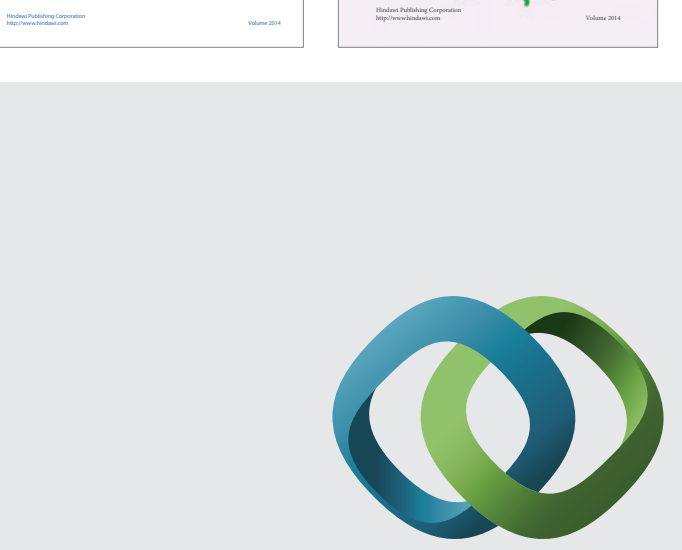

\section{Hindawi}

Submit your manuscripts at

http://www.hindawi.com
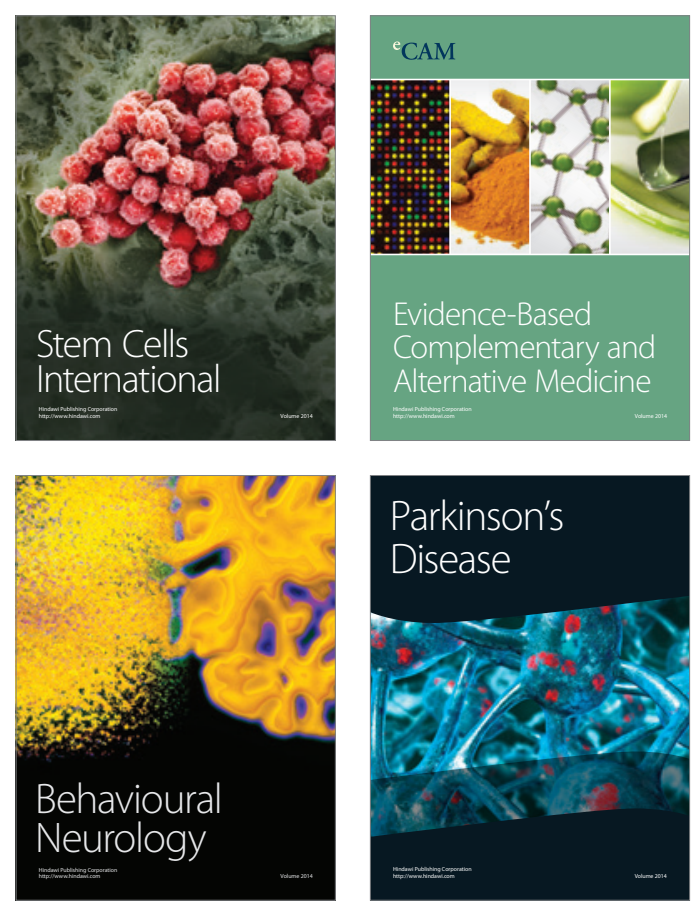

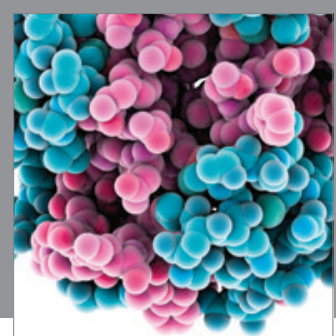

Journal of
Diabetes Research

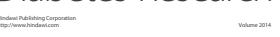

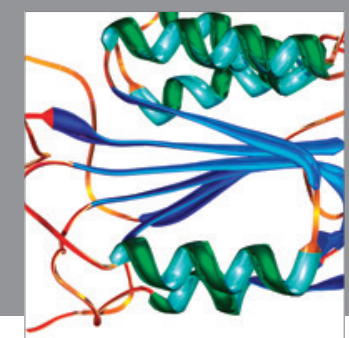

Disease Markers
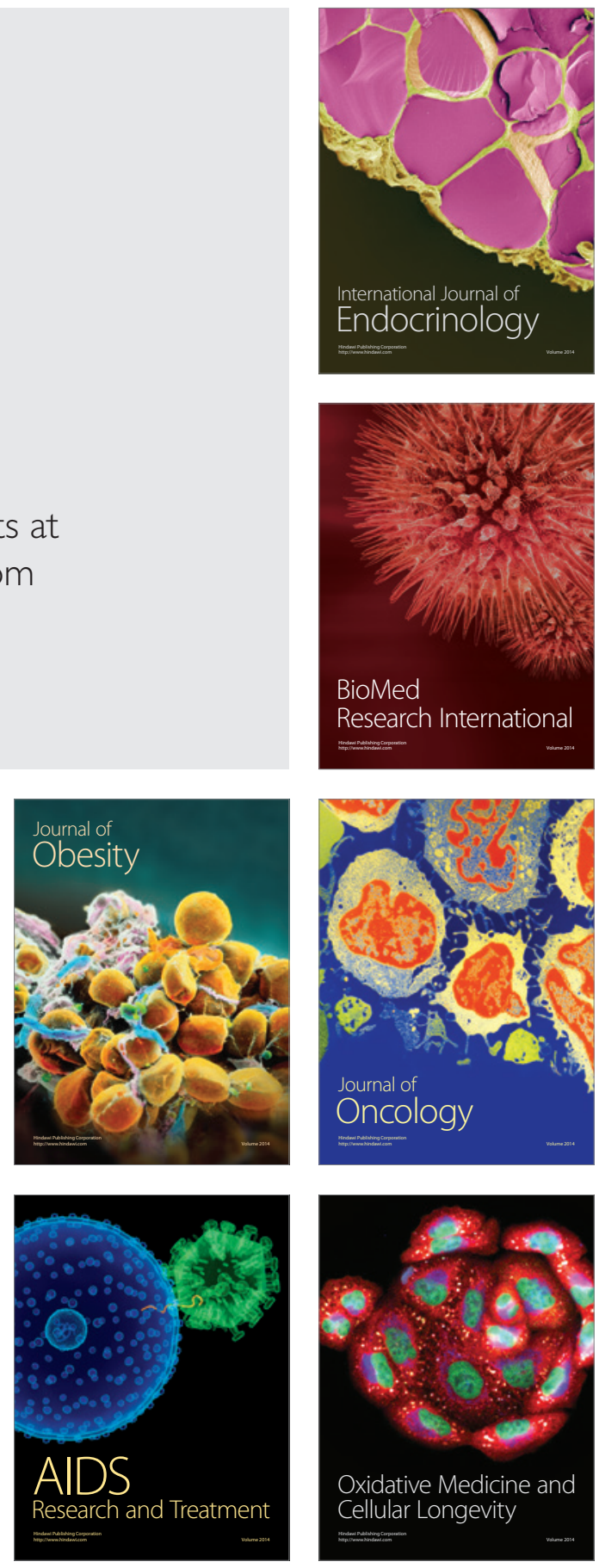